\title{
Éditorial
}

\section{La décentralisation}

Le débat sur la décentralisation gouvernementale revient à l'agenda politique cet automne 2003 alors que le nouveau gouvernement désire consulter les élus en régions avant de revoir les modalités de gestion publique de l'État québécois. Déjà, la réflexion est commencée ici et là. On l'active d'abord dans plusieurs agences publiques centrales comme les ministères, commissions, comités et offices. Ensuite, les agences plus ou moins décentralisées telles que les corporations, directions, conseils et bureaux se préparent au débat. Et finalement, on réfléchit à cet enjeu de la décentralisation dans les regroupements nationaux des multiples acteurs locaux, MRC et régionaux qui ouvrent dans divers secteurs d'activités publics tels que la santé, l'éducation, la culture, le développement. D'une manière disjointe, on élabore des diagnostics, on soulève des pronostics et on formule évidemment des recommandations sous la forme de thérapeutiques.

Les aviseurs, conseillers, démarcheurs et autres défenseurs d'opinions et d'intérêts spécifiques sont nombreux dans le vaste secteur public. Généralement, les positions réciproques divergent aussi de manière considérable. Un tel contexte de compétition entre divers intérêts corporatistes bien défendus possède certes des vertus, notamment celle d'éclairer convenablement le législateur. Il génère cependant un certain nombre de vices. Parmi ces derniers, il va sans dire que le ralentissement ou le freinage du processus décisionnel national est sûrement le plus néfaste en considérant la réforme locale et régionale jugée nécessaire.

Car la difficulté de prendre une décision satisfaisante face à tous les intérêts opposés et défendus auprès du réformateur explique largement pourquoi la réforme locale et régionale a piétiné, multiplié les

Paul Prévost

Université de Sherbrooke retards et avancé très lentement au cours des quatre dernières décennies largement marquées par la bonne volonté réformatrice des gouvernements successifs. C'est ce qui explique en grande partie le désir très actuel d'un retour en arrière dans le dossier des fusions municipales. Par contre, des éléments concrets de réforme ont fait progresser considérablement les affaires locales et régionales au Québec. Pensons notamment à la réforme de la fiscalité municipale, à la Loi sur l'aménagement et l'urbanisme, au décret sur l'uniformisation des territoires en 1988, à la création des centres locaux de développement et, évidemment, à la réforme des agglomérations urbaines. De cette trajectoire pavée de succès, d'échecs et de demi-succès dans la réforme locale et régionale, nous pouvons retenir un certain nombre de conditions qui facilitent le législateur, notamment la présence active d'un table interministérielle de travail au sein du Conseil exécutif.

Selon une perspective locale et régionale, le grand nombre et la diversité des intérêts en cause plaident pour l'élaboration collective d'une position commune globale à soumettre aux autorités gouvernementales de Québec. Une telle position pouvant faire consensus deviendrait le chien de garde du cheminement réformateur du gouvernement. À cet effet de position commune, il revient aux différents acteurs des milieux locaux et régionaux concernés d'instaurer un Forum québécois des territoires afin de préparer cette position commune articulée dans un esprit de respect des intérêts de chaque partenaire. Les modalités organisationnelles d'un tel mécanisme de concertation nationale de la société civile organisée aux échelles territoriales sont à définir par les partenaires à associer dès le départ. À notre avis, le leadership initial doit nécessairement venir des fédérations municipales, locales et régionales actives à l'échelle québécoise.

Marc-Vrbain Proufx

Université du Québec à Chicoutimi 